\title{
The Islamic Guidance in Counselling: An Overview
}

\author{
M.M.A. Abdullah*1 and S.M.M. Mazahir ${ }^{2}$ \\ ${ }^{1 \& 2}$ Department of Islamic Studies, South Eastern University of Sri Lanka
}

\begin{abstract}
Counselling is a professional process that provides guidance for human beings to face various psychological problems successfully. Islam guides all aspects of human life and provides various guidelines for dealing with psychological challenges. Hence, the study was conducted to identify the theory and practical Islamic guidelines on counselling. Based on the earlier literature, the information on counselling in the Islamic basic sources, the al-Qur'an and al-Hadith along with research articles, books, web articles were analyzed. The main finding of this study is that Islam has provided practical guidelines for overcoming psychological problems. This article would help understand the value and the importance of Islamic counselling.
\end{abstract}

Keywords: Islamic Counselling, Islam, Counselling, Religious Counselling, Al-Qur'an, Al-Sunnah, AlHadith.

*Corresponding Author Email: abdullahmma416@gmail.com

\section{உளவளத்துணை தொடர்பான இஸ்லாமிய வழிகாட்டல்கள்: ஓர் நநாக்கு}

எம். எம். ஏ. அப்துல்லாஹ் ${ }^{1}$ மற்றும் எஸ். எம். எம். மஸாஹிர் ${ }^{2}$

1\&2இஸ்லாமிய கற்கைகள் துறை, இலங்கை தென்கிழக்குப் பல்கலைக்கழகம்

\section{ஆய்வுச் சுருக்கம் (Abstract)}

மனிதன் எதிர்கொள்ளும் பல்வேறு உளவியல் ரீதியான பிரச்சினைகளை வெற்றிகரமாக எதிர்கொள்வதற்கான வழிகாட்டல்களை வழங்கும் தொழில்முறைசார்ந்த செயற்பாடே உளவளத்துணையாகும். மனித வாழ்வின் அனைத்துத் துறைகளுக்குமான வழிகாட்டல்களை வழங்கும் இஸ்லாம், உளவியல் ரீதியான சவால்களை எதிர்கொள்வதற்கான பல்வேறு வழிகாட்டல்களை வழங்கியுள்ளது. அந்தவகையில் உளவளத்துணை தொடர்பான கோட்பாடு மற்றும் நடைமுறை சார்ந்த இஸ்லாமிய வழிகாட்டல்களை இனங்காணும் நோக்கில் இவ்வாய்வு மேற்கொள்ளப்பட்டது. முன்னைய இலக்கியங்களை மையப்படுத்தி மேற்கொள்ளப்பட்ட இவ்வாய்வுக்காக இஸ்லாமிய மூல ஆவணங்களான அல்குர்ஆன் மற்றும் அஸ்ஸ்ன்னா என்பவற்றுடன் ஆய்வுக் கட்டுரைகள், நூல்கள், இணையத்தள ஆக்கங்கள் போன்றவற்றில் காணப்படும் உளவளத்துணை தொடர்பான தகவல்கள் பகுப்பாய்வுக்குட்படுத்தப்பட்டுள்ளன. மனிதன் எதிர்கொள்ளும் உளவியல் சார் பிரச்சினைகளை வெற்றிகரமாக எதிர்கொள்வதற்கான நடைமுறைச் சாத்தியமான பல்வேறு வழிகாட்டல்களை இஸ்லாம் வழங்கியுள்ளது என்பதே இவ்வாய்வின் பிரதான கண்டறிதலாகும். இஸ்லாமிய உளவளத்துணையின் பெறுமானம் மற்றும் அதன் அவசியம் தொடர்பில் அறிந்துகொள்வதற்கு இவ்வாய்வு உறுதுணையாக அமையவல்லது.

திறவுச் சொற்கள்: இஸ்லாமிய உளவளத்துணை, இஸ்லாம், உளவளத்துணை, சமயம்சார் உளவளத்துணை, அல்குர்ஆன், அஸ்ஸுன்னா, அல்ஹதீஸ். 


\section{அறிமுகம் (Introduction)}

நவீன உலகில் மனிதன் எதிர்கொள்ளும் பல்வேறு பிரச்சினைகளின் விளைவாக மன அழுத்தம், மனச் சோர்வு, பதற்றம் போன்ற பல்வேறு உளப்பிரச்சினைகளுக்கு அவன் உள்ளாகிறான். இவ்வாறான உளவியல் பிரச்சினைகள் தீர்க்கப்படுவதற்கான வழிகளுள் மதம்சார் ஆற்றுப்படுத்தல் (Religious Counselling) மிக முக்கியமான ஒன்றாகும் [1]. ஏனெனில் மனித வாழ்வில் மனித உள்ளம் ஆரோக்கியமானதாக மாறுவற்கு உடல் மற்றும் ஆன்மீக ரீதியான தேவைகளுக்கிடையில் சமத்துவம் பேணப்படுவது மிகவும் அவசியமானதாகும். அவ்வாறு செய்யத் தவறும் போது மனித வாழ்வு பல்வேறு பின்னடைவுகளையும் தோல்விகளையும் சந்திக்கிறது [2].

இன்று பரவலாக நடைமுறையிலிருக்கும் உளவளத்துணைக் கோட்பாடுகள் மற்றும் சிகிச்சை முறைகள் மேலைத்தேய அறிஞர்களின் மூலம் உருவாக்கப்பட்டவையாகும். இஸ்லாமிய நாடுகள் மற்றும் ஏனைய நாடுகளில் வாழும் முஸ்லிம் உளவளத்துணையாளர்களில் அதிகமானோர் தமது சேவைநாடிகளுக்கு உளவளத்துணையினூடாக சிகிச்சை அளிக்கும் போதும், உளவளத்துணைக் கோட்பாடுகளைக் கற்பிக்கும் போதும் மேலைத்தேய கோட்பாடுகள் மற்றும் நடைமுறைகளை ஒட்டியே அவை மேற்கொள்ளப்படுகின்றன [3].

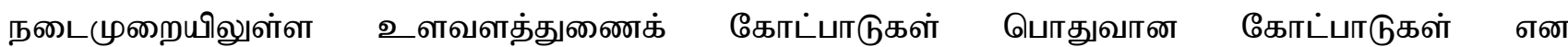
அடையாளப்படுத்தப்படினும் கூர்ந்து நோக்குமிடத்து அவை மேலைத்தேயக் கோட்பாடுகள் என்பதை எம்மால் அவதானிக்க முடியும். ஏனெனில் அவைகளில் பெரும்பாலானவை மேலைத்தேய அறிஞர்களால் உருவாக்கப்பட்டவையாகும் [4].

மேலைத்தேய உளவளத்துணைக் கோட்பாடுகளின் செல்வாக்கின் விளைவா இஸ்லாமிய உளவளத்துணை வழிகாட்டல்கள் தொடர்பில் மிகவும் குறைவாகவே அவதானம் செலுத்தப்படுகிறது [5]. இஸ்லாத்தின் அடிப்படை மூலாதாரங்களான அல்குர்ஆன் மற்றும் அல்ஹதீஸின் போதனைகளை ஆராயும் போது உளவியல் மற்றும் உளவளத்துணை பற்றிய வழிகாட்டல்கள் தொடர்பிலான அதன் புரிதலை மிகத் தெளிவாக விளங்கிக் கொள்ள முடியும் [6]. இவை தெய்வீகத்தன்மை கொண்டவையாக இருப்பதனால் மனித உடல், உள்ளம் மற்றும் ஆன்மா தொடர்பில் மிகத் தெளிவான நிலைப்பாட்டை கொண்டுள்ளதோடு அவற்றுக்கு மத்தியில் மிகத் துல்லியமான சமநிலையையும் பேணுகின்றன [2 \& 7].

மேலைத்தேய உளவளத்துணைக் கோட்பாடுகள் மனிதனின் உள அமைதிக்கு மனிதனின் லௌகீகத் தேவைகளை நிறைவேற்றுவதையும் பிறரோடு நல்ல முறையில் நடந்து கொள்ளல் போன்ற விடயங்களையும் மாத்திரமே மையப்படுத்தி அமைந்துள்ளன [8]. எனினும் இஸ்லாமிய உளவளத்துணை ஆன்மீகத்துக்கும் மனிதனின் உள அமைதிக்கும் நெருங்கிய தொடர்பை பேணுகிறது [9]. மனிதனின் ஆன்மீக ஈடுபாடே உள அமைதியின் அடிப்படையாகும். எனினும் மனிதனின் உலக, உடலியல் தேவைகளையும் கவனத்திற் கொள்ளுமாறும் இஸ்லாம் வலியுறுத்துகின்றது [10].

இது பற்றி அல்குர்ஆன் " “மேலும், அல்லாஹ் உனக்குக் கொடுத்த (செல்வத்)திலிருந்து மறுமை வீட்டைத் தேடிக் கொள். எனினும், இவ்வுலகத்தில் உன் பங்கை (உனக்கு விதித்திருப்பதையும்) மறந்து விடாதே! அல்லாஹ் உனக்கு நல்லதைச் செய்திருப்பதைப் போல், நீயும் நல்லதை செய்! இன்னும், பூமியில் குழப்பம் செய்ய விரும்பாதே. நிச்சயமாக அல்லாஹ் குழப்பம் செய்பவர்களை நேசிப்பதில்லை" (28:77) எனக் குறிப்பிடுகிறது.

அந்தவகையில் இவ்வாய்வானது ஆற்றுப்படுத்தல் தொடர்பான இஸ்லாமிய நோக்கினை அதன் மூல ஆவணங்களான அல்குர்ஆன் மற்றும் நபி (ஸல்) அவர்களிள் நடைமுறை வழிகாட்டலான அஸ்ஸீன்னாவின் துணைகொண்டு விபரிக்க முனைகிறது.

\section{ஆய்வுப் பிரச்சினை (Research Problem)}

இஸ்லாம் மனித வாழ்வின் அனைத்துத் துறைகளுக்குமான அழகிய வழிகாட்டல்களை வழங்கும் வாழ்க்கை நெறியாகும் [11]. அன்றாடம் மனிதன் எதிர்கொள்ளும் உள ரீதியான பிரச்சினைகளுக்கான 
நடைமுறைச் சாத்தியமான பல்வேறு வழிகாட்டல்கள் இஸ்லாமிய மூல ஆவணங்களான அல்குர்ஆன் மற்றும் அல்ஹதீஸினூடாக வழங்கப்பட்டுள்ளன. நவீன உளவளத்துணைச் செயன்முறைகளில் மேற்கத்தேய உளவளத்துணைக் கோட்பாடுகளின் செல்வாக்கு காணப்படுகின்ற போதும் இஸ்லாத்தின் அடிப்படை மூலாதாரங்களும் மனிதன் அன்றாடம் எதிர்கொள்ளும் பல்வேறு உளவியல் சார் பிரச்சினைகளை எதிர்கொள்வதற்கான சீரிய வழிகாட்டல்களை வழங்கியுள்ளன என்பதை ஆய்வுப் பிரச்சினையாகக் கொண்டு இந்த ஆய்வு மேற்கொள்ளப்பட்டுள்ளது.

\section{ஆய்வு நோக்கம் (Research Objective)}

இவ்வாய்வு உளவளத்துணை தொடர்பான கோட்பாடு மற்றும் நடைமுறை சார்ந்த இஸ்லாமிய வழிகாட்டல்களைக் கண்டறிவதை நோக்காகக் கொண்டுள்ளது.

\section{ஆய்வு முறையியல் (Research Methodology)}

முன்னைய இலக்கியங்களை மையப்படுத்தி மேற்கொள்ளப்பட்ட இவ்வாய்வுக்காக இஸ்லாமிய மூல ஆவணங்களான அல்குர்ஆன் மற்றும் அல்ஹதீஸ் என்பவற்றுடன் ஆய்வுக் கட்டுரைகள், நூல்கள், இணைத்தள் ஆக்கங்கள் மற்றும் ஏனைய இலக்கியங்களில் காணப்படும் உளவளத்துணை தொடர்பான தகவல்கள் பகுப்பாய்வுக்கு உட்படுத்தப்பட்டு, முடிவுகள் முன்வைக்கப்பட்டுள்ளன.

\section{பெறுபேறுகளும் கலந்துரையாடலும் (Results and Discussion)}

\section{1. இஸ்லாமிய உளவளத்துணை மற்றும் மார்க்கப் போதனை (பயான்) ஆகியவற்றுக்கிடையிலான வேறுபாடு}

மார்க்கப் போதனைகள் மற்றும் இஸ்லாமிய உளவளத்துணை ஆகியவற்றுக்கிடையில் குறிப்பிடத்தக்களவு வேறுபாடுகள் நிலவுகின்றன. மார்க்கப் போதனைகள் தனி நபருக்கான அல்லது ஒரு குழுவுக்கான அல்குர்ஆன் மற்றும் அஸ்ஸீன்னாவின் போதனைகளை கற்பித்து அவற்றினடிப்படையில் வாழ்வை அமைத்துக் கொள்வதற்கான வழிகாட்டல்களை வழங்கும் செயற்பாடாகும். பெரும்பாலும் அச்செயற்பாடு ஒரு தரப்பை (மார்க்க அறிஞரை) மையப்படுத்தியதாகும். ஒழுங்கமைக்கப்பட்ட சமயம் சார் தகவல்களை நோக்கி பிறரை வழிநடாத்துவதே இதன் பிரதான இலக்காகும் [12]. இஸ்லாமிய உளவளத்துணையை பொறுத்தவரை சமநிலையான மனோநிலையைத் தோற்றுவித்தல் தொடர்பிலேயே அது கவனம் செலுத்துகிறது. இச்செயன்முறையில் சேவை நாடியின் நடத்தை இஸ்லாமிய சமய நம்பிக்கைகளுடனும் போதனைகளுடனும் ஒன்றியதாகவே அமையப்பெற்றிருக்கும். இதனால் அவனது ஆளுமை மற்றும் சுபீட்சம் என்பவற்றுக்கிடையில் சமநிலை பேணப்படுவதோடு அவனது உள்ளமும் ஆரோக்கியமானதாக மாறிவிடுகிறது. அத்துடன் இச்செயற்பாடு ஆற்றுப்படுத்துனர் மற்றும் சேவைநாடி ஆகிய இருவரையும் மையப்படுத்தியதாகும் [13].

\section{2. இஸ்லாமிய உளவளத்துணையில் அல்குர்ஆன் மற்றும் அஸ்ஸுன்னாவின் வகிபாகம்}

இஸ்லாமிய உளவளத்துணையில் பிரதான மூலாதாரங்களாக அல்குர்ஆன் மற்றும் அல்ஹதீஸ் ஆகியவையே திகழ்கின்றன [14]. ஆல்குர்ஆனில் பல்வேறு இடங்களில் உளவளத்துணை தொடர்பான பல்வேறு வழிகாட்டல்கள் இடம்பெற்றுள்ளன [15].

Sudan (2017) [14] என்பவர் “மனிதர்களே! உங்கள் இறைவனிடமிருந்து உங்களுக்கு நிச்சயமாக ஒரு நல்லுபதேசமும் வந்துள்ளது. (உங்கள்) இதயங்களிலுள்ள நோய்களுக்கு அருமருந்தும் (வந்திருக்கிறது) மேலும் (அது) மு. ‘மின்களுக்கு நேர்வழிகாட்டியாகவும், நல்லருளாகவும் உள்ளது” (10:57) மற்றும் “(நேர் வழி பெறும்) அவர்கள் எத்தகையோரென்றால், அவர்கள் தாம் (முற்றிலும்) ஈமான் கொண்டவர்கள். மேலும், அல்லாஹ்வை நினைவு கூறுவதால் அவர்களுடைய இதயங்கள் அமைதி பெறுகின்றன. அல்லாஹ்வை நினைவுகூறுவது கொண்டு தான் இதயங்கள் அமைதி பெறுகின்றன என்பதை அறிந்து கொள்க!" (04:170) போன்ற அல்குர்ஆனிய வசனங்களை அடிப்படையாகக் கொண்டே இஸ்லாமிய உளவளத்துணை தோற்றம் பெற்றிருப்பதாக விபரிக்கிறார். 
அதேபோல் Mursy மற்றும் Rasheedi (1988) [16] ஆகியோர் இஸ்லாமிய மூலாதாரங்களான அல்குர்ஆன் மற்றும் அஸ்ஸுன்னாவை அடிப்படையாகக் கொண்ட உளவளத்துணைச் செயன்முறையின் பிரதான அடிப்படைகளாக பின்வருவனவற்றைக் குறிப்பிடுகின்றனர்.

i. பிறர் நலன் நாடல். இதனையே நபி (ஸல்) அவர்கள் “மார்க்கம் என்பது பிறர் நலன் நாடுவதாகும்” (முஸ்லிம், 55) எனக் குறிப்பிட்டார்கள்.

ii. பிறருக்கான வழிகாட்டல்களை வழங்குவது இறைவனிடம் மிகுந்த வெகுமதியைப் பெற்றுத்தரும் நற்செயலாகும். ஏனெனில் இதனூடாக பலர் நன்மையடைவர். இதனையே நபி (ஸல்) அவர்கள் “ஒரு அடியான் தனது சகோதரனுக்கு உதவும் காலமெல்லாம் இறைவன் அவனுக்கு உதவுகிறான்" (முஸ்லிம், 2699) எனக் குறிப்பிட்டார்கள்.

iii. ஒருவர் தனக்குப் பயன்தரும் விடயங்களில் ஈடுபட்டு பயனற்ற விடயங்களைத் தவிர்ந்து கொள்ளல். இதனையே நபி (ஸல்) அவர்கள் “உனக்குப் பயன்தரும் விடயங்களை ஆவல் கொள். இறைவனிடம் உதவி தேடு. நீ நம்பிக்கையிழந்து விடாதே!" (முஸ்லிம், 2664) எனக் குறிப்பிட்டார்கள்.

iv. ஒவ்வொருவரும் இறைவன் அனுமதித்த வரையறைகளுக்கு உட்பட்டு சுயமாக முடிவுகளை எடுப்பதற்கும், தனக்குப் பொருத்தமாவற்றைத் தேர்ந்தெடுத்துக் ொள்வற்கும் உரிமையுடையவராவார். இதனையே “அவர்கள் சம்பாதித்தவை அவர்களுக்கே. நீங்கள் சம்பாதித்தவை உங்களுக்கே!" (2:134) எனும் வசனம் வலியுறுத்துகிறது.

\section{3. அல்குர்ஆன் மற்றும் அஸ்ஸுன்னாவை அடிப்படையாகக் கொண்ட ஆற்றுப்படுத்தல் செயன்முறையின் இலக்குகள்}

அல்குர்ஆன் மற்றும் அஸ்ஸீன்வை அடிப்படையாகக் கொண்ட ஆற்றுப்படுத்தல் செயன்முறை பின்வரும் இலக்குகளைக் கொண்டமைந்துள்ளது [13].

\section{I. தனி மனிதனுக்குள் ஏற்படும் உளப்போராட்டங்களில் இருந்து அவனை விடுவித்தல்}

மனிதனுக்குள் பல்வேறு உளப்போராட்டங்கள் இருப்பது இயல்பானதாகும் [17]. இதனையே நபி (ஸல்) அவர்கள்,
“ஒவ்வொரு மனிதரையும் (மனித உள்ளத்தையும்) ஷைத்தான் தீண்டுகிறான். அவ்வாறே மலக்கும் (வானவரும்) தீண்டுகிறார். (உள்ளத்துக்குள்) மோசமானவற்றையும், உண்மையை மறுக்குமாறும் தூண்டுவதே ஷைத்தானின் தீண்டலாகும். நல்லவற்றையும், சத்தியத்தை ஏற்றுக்கொள்ளும்படியும் தூண்டுவதே மலக்கின் தீண்டலாகும். யார் அதனை (மலக்கின் தீண்டலை) உணர்கிறாரோ அவர் இறைவனைப் புகழட்டும். யார் மற்றதை (ஷைத்தானின் தீண்டலை) உணர்கிறாரோ அவர் ஷைத்தானிடமிருந்து இறைவனிடம் பாதுகாவல் தேிக் கொள்ளட்டும். பின்னர் நபி (ஸல்) அவர்கள் “(தான தர்மங்கள் செய்வதினால்) வறுமை (உண்டாகிவிடும் என்று அதைக்) கொண்டு உங்களை ஷைத்தான் பயமுறுத்துகிறான். ஒழுக்கமில்லாச் செயல்களைச் செய்யுமாறும் உங்களை ஏவுகிறான். ஆனால் அல்லாஹ்வோ, (நீங்கள் தான தருமங்கள் செய்தால்) தன்னிடமிருந்து மன்னிப்பும், (அருளும், பொருளும்) மிக்க செல்வமும் (கிடைக்கும் என்று) வாக்களிக்கிறான்" (2: 268) எனும் வசனத்தை ஓதிக் காண்பித்தார்கள்" (திர்மிதி, 2988) எனும் நபிமொழியினூடாகத் தெளிவுபடுத்தினார்கள்.

\section{II. பகுத்தறிவுக்கு அ அப்பாற்பட்ட சிந்தனைகளை வெற்றிகரமாக எதிர்கொள்வதற்குத் தேவையான பயிற்சிகளை வழங்கல்}

இறைவன் வானங்கள், பூமி உட்பட இப்பிரபஞ்சத்திலுள்ள ஏனைய படைப்பினங்களின் ஊடாக தனது ஆற்றலையும் மகத்துவத்தையும் தெளிவுபடுத்தியுள்ளான். இவையனைத்தும் இறைவனின் நுட்பமான, சீரிய, ஒப்பற்ற ஆற்றலுக்கான சிறந்த எடுத்துக்காட்டுகளாகும் [13]. இதனையே இறைவன்,"(நபியே!) நீர் கூறுவீராக: “எல்லாப் புகழும் அல்லாஹ்வுக்கே உரியது. இன்னும் அவன் தேர்ந்தெடுத்துக் கொண்ட அவனுடைய 
அடியார்கள் மீது ஸலாம் உண்டாவதாக! அல்லாஹ் மேலானவனா? அல்லது அவர்கள் (அவனுக்கு) இணையாக்குபவை (மேலானவை)யா?" இந்தப் பூமியை வசிக்கத் தக்க இடமாக ஆக்கியவனும், அதனிடையே ஆறுகளை உண்டாக்கியவனும், அதற்காக (அதன் மீது அசையா) மலைகளை உண்டாக்கியவனும், இரு கடல்களுக்கிடையே தடுப்பை உண்டாக்கியவனும் யார்? அல்லாஹ்வுடன் (வேறு) நாயன் இருக்கிறானா? இல்லை! (எனினும்) அவர்களில் பெரும்பாலோர் அறியாதவர்களாக இருக்கின்றனர். கஷ்டத்திற்குள்ளானவன் அவனை அழைத்தால் அவனுக்கு பதில் கொடுத்து, அவன் துன்பத்தை நீக்குபவனும், உங்களை இப்பூமியில் பின்தோன்றல்களாக ஆக்கியவனும் யார்? அல்லாஹ்வுடன் (வேறு) நாயன் இருக்கின்றானா? (இல்லை). எனினும் (இவையெல்லாம் பற்றி) நீங்கள் சிந்தித்துப் பார்ப்பது மிகக் குறைவேயாகும். கரையிலும் கடலிலுமுள்ள இருள்களில் உங்களை நேரான வழியில் செலுத்துபவன் யார்? மேலும், தன்னுடைய “ரஹ்மத்” என்னும் அருள் மாரிக்கு முன்னே நன்மாராயம் (கூறுவன) ஆக காற்றுகளை அனுப்பி வைப்பவன் யார்? அல்லாஹ்வுடன் (வேறு) நாயன் இருக்கின்றானா? அவர்கள் இணை வைப்பவற்றை விட அல்லாஹ் மிகவும் உயர்வானவன்" (27:59-63) எனும் வசனங்களின் ஊடாகத் தெளிவுபடுத்துகிறான்.

\section{III. விழிப்புணர்வை அதிகரித்தல்}

இஸ்லாமிய உளவளத்துணையின் இ இலக்குகளுள் தனிமனித அறிவியல் விழிப்புணர்வும் முக்கியமானதாகும் [17]. அல்குர்ஆனின் பல்வேறு வசனங்கள் இவ்விடயத்தை வலியுறுத்துகின்றன. எடுத்துக்காட்டாக

"பூமியில் பிராயாணம் செய்து, குற்றவாளிகளின் முடிவு என்னவாயிற்று என்று பாருங்கள் என்று (அவர்களிடம் நபியே!) நீர் கூறுவீராக. அவர்களுக்காக நீர் கவலைப்படாதீர். மேலும், அவர்கள் செய்யும் சூழ்ச்சியைப் பற்றியும் நீர் சங்கடத்தில் ஆக வேண்டாம். இன்னும் “நீங்கள் உண்மை கூறுபவர்களாக இருந்தால் (வேதனை பற்றிய) இந்த வாக்குறுதி எப்பொழுது (நிறைவேறும்?)" என்றும் அவர்கள் கேட்கிறார்கள். "நீங்கள் அவசரப்படுபவற்றில் சில இப்பொழுதே உங்களுக்கு வந்து சேரக்கூடும்” என்று (நபியே!) நீர் கூறிவிடுவீராக. இன்னும் நிச்சயமாக உம் இறைவன் மனிதர்கள் மீது மிக்க கிருபையுடையவனாகவே இருக்கின்றான். ஆனால் அவர்களில் பெரும்பாலோர் நன்றி செலுத்துவதில்லை. மேலும் அவர்களின் இருதயங்கள் மறைத்து வைத்திருப்பதையும், அவர்கள் வெளிப்படுத்துவதையும் நிச்சயமாக உம் இறைவன் நன்கறிவான். வானத்திலும், பூமியிலும் மறைந்துள்ளவற்றில் எதுவும் (லவ்ஹல் மஹ்.'பூள் எனும்) தெளிவான குறிப்பேட்டில் பதிவு செய்யப்படாமல் இல்லை. நிச்சயமாக இந்த குர்ஆன் பனூ இஸ்ராயீல்களுக்கு அவர்கள் கருத்து வேறுபாடு கொண்டிருந்ததில் பெரும்பாலானதை விவரித்துக் கூறுகிறது" (27:69-76) ஆகிய வசனங்களைக் குறிப்பிட முடியும்.

\section{IV. கடந்த காலம் தொடர்பான சிந்தனைகளைத் குறைத்து, நிகழ் காலம் தொடர்பான விடயங்களில் அதிக கவனத்தைச் செலுத்துதல்.}

ஒரு மனிதன் தனது கடந்த காலம் தொடர்பான சிந்தனைகளைத் குறைத்து, நிகழ்காலம் தொடர்பான விடயங்களில் அதிக கவனத்தைச் செலுத்தும் மனோநிலையைத் தோற்றுவிக்கின்றமை அல்குர்ஆன் மற்றும் அஸ்ஸுன்னாவின் அடிப்படையிலமைந்த ஆற்றுப்படுத்தல் செயன்முறையின் இலக்குகளுள் முக்கியமான ஒன்றாகும் [13].

இதனையே “(நினைவு கூறுங்கள்! உஹது களத்தில்) உங்கள் பின்னால் இ இருந்து இறைதூதர் உங்களை அழைத்துக் கொண்டிருக்க, நீங்கள் எவரையும் திரும்பிப் பார்க்காமல் மேட்டின் மேல் ஏறிக் கொள்ள ஓடிக் கொண்டிருந்தீர்கள். ஆகவே, (இவ்வாறு இறை தூதருக்கு நீங்கள் கொடுத்த துக்கத்தின்) பலனாக இறைவன் துக்கத்தின் மேல் துக்கத்தை உங்களுக்குக் கொடுத்தான். ஏனெனில் உங்களுக்குக் கிடைக்க வேண்டியது தவறி விட்டாலோ, உங்களுக்குச் சோதனைகள் ஏற்பட்டாலோ நீங்கள் (சோர்வும்) கவலையும் அடையாது (பொறுமையுடன் இருக்க வேண்டும் என்பதற்காகத்தான்).

இன்னும், அல்லாஹ் நீங்கள் செய்வதை நன்கு அறிபவனாக இருக்கின்றான்" (03:153) எனும் அல்குர்ஆனிய வசனம் தெளிவுபடுத்துகிறது. 


\section{வெற்றி எனும் இலக்கை நோக்கிய நகர்வு}

அல்குர்ஆன் மற்றும் அஸ்ஸுன்னாவின் அடிப்படையிலமைந்த ஆற்றுப்படுத்தல் செயன்முறையின் இலக்குகளுள் வெற்றியை நோக்கிய நகர்வும் ஒன்றாகும். இஸ்லாமிய மூல ஆவணங்கள் வெற்றியை வரையறை செய்து அவற்றை அடையும் முறை தொடர்பில் மிகத் தெளிவான வழிகாட்டல்களை வழங்குகின்றன [13].

எடுத்துக்காட்டாக “நீ எங்களை நேர்வழியில் நடத்துவாயாக!” (1: 6) எனும் அல்குர்ஆனிய வசனம் வெற்றியை அடைவதற்கான அவசியத்தை வலியுறுத்துவதோடு “(அது) நீ எவர்களுக்கு அருள் புரிந்தாயோ அவ்வழி. (அது) உன் கோபத்திற்கு ஆளானோர் வழியுமல்ல. நெறி தவறியோர் வழியுமல்ல" (1:7) எனும் வசனம் நேரான பாதையிலிருந்து விலகிச் சென்று தோல்வியை நோக்கி நகர்வதை எச்சரிக்கிறது.

\section{V. பொறுப்புணர்வை அதிகரித்தல்}

பொறுப்புணர்வு பற்றிய விழிப்புணர்வை அதிகரிப்பதும் அல்குர்ஆன் மற்றும் அஸ்ஸுன்னாவின் அடிப்படையிலமைந்த ஆற்றுப்படுத்தல் செயன்முறையின் இலக்குகளுள் ஒஒன்றாகும். இதனையே “எனவே, எவர் ஓர் அணுவளவு நன்மை செய்திருந்தாலும் அத(ற்குரிய பல)னை அவர் கண்டு கொள்வார். அன்றியும், எவன் ஓர் அணுவளவு தீமை செய்திருந்தாலும், அ(தற்குரிய பல)னையும் அவன் கண்டு கொள்வான்" (99: 7-8) ஆகிய வசனங்கள் தெளிவுபடுத்துகின்றன [17].

\section{VI. மனித வாழ்வுக்கான இலட்சியத்தை உருவாக்கல்}

அல்குர்ஆன் மற்றும் அஸ்ஸுன்னாவின் அடிப்படையிலமைந்த ஆற்றுப்படுத்தல் செயன்முறை மனிதப் படைப்பின் நோக்கத்தை வலியுறுத்தி, மனித வாழ்வுக்கான இலட்சியத்தை உருவாக்குவதையே தனது முக்கிய இலக்காகக் கொண்டுள்ளது [17].

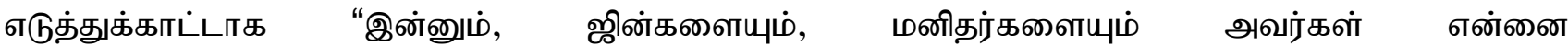
வணங்குவதற்காகவேயன்றி நான் படைக்கவில்லை. அவர்களிடமிருந்து எந்தப் பொருளையும் நான் விரும்பவில்லை. எனக்கு அவர்கள் உணவு அளிக்க வேண்டுமென்றும் நான் விரும்பவில்லை. நிச்சயமாக அல்லாஹ்தான் உணவு அளித்துக் கொண்டிருப்பவன். பலம் மிக்கவன். உறுதியானவன்" (51:56-58) ஆகிய வசனங்கள் இறைவழிபாடே மனித இன உருவாக்கத்தின் அடிப்படை நோக்கம் எனும் கருத்தை தெளிவுபடுத்துகின்றன.

அதேபோல்

“(நபியே) இன்னும், உம் இறைவன் வானவர்களை நோக்கி “நிச்சயமாக நான் பூமியில் ஒரு பிரதிநிதியை அமைக்கப்போகிறேன்" என்று கூறியபோது, அவர்கள் “(இறைவா!) நீ அதில் குழப்பத்தை உண்டாக்கி, இரத்தம் சிந்துவோரையா அமைக்கப்போகிறாய்? இன்னும் நாங்களோ உன் புகழ் ஓதியவர்களாக உன்னைத் துதித்து, உன் பரிசுத்தத்தைப் போற்றியவர்களாக இருக்கின்றோம் என்று கூறினார்கள். அ(தற்கு இறை)வன் "நீங்கள் அறியாதவற்றையெல்லாம் நிச்சயமாக நான் அறிவேன்" எனக் கூறினான்" (02:30)

மற்றும்

“நாம் உங்களைப் படைத்ததெல்லாம் வீணுக்காக என்றும், நீங்கள் நம்மிடத்தில் நிச்சயமாக மீட்டப்படமாட்டீர்கள் என்றும் எண்ணிக் கொண்டீர்களா?" (என்றும் இறைவன் கேட்பான்). ஆகவே, உண்மையில் அரசனான அல்லாஹ், மிக உயர்ந்தவன். அவனைத் தவிர நாயனில்லை. கண்ணியமிக்க அர்ஷின் இறைவன் அவனே!" (23: 115-116) ஆகிய வசனங்களும் மனித வாழ்வின் இலட்சியத்தை நோக்கிய பயணத்தின் அவசியத்தை உணர்த்துகின்றன. 


\section{VII. மனித உள்ளத்தை கட்டுப்படுத்துவதற்கு உதவுதல்}

தனிமனிதர்கள் தமது உள்ளத்தை கட்டுப்பாட்டுக்குள் வைத்துக்கொள்வற்குத் தேவையான உதவிகளை வழங்குவதும் அல்குர்ஆன் மற்றும் அஸ்ஸுன்னாவின் அடிப்படையிலமைந்த ஆற்றுப்டுத்தல் செயன்முறையின் இலக்குகளுள் ஒன்றாகும் [17].

இதனையே “மு.:மின்களே! பொறுமையுடன் இருங்கள். (இன்னல்களை) சகித்துக் கொள்ளுங்கள். (ஒருவரை ஒருவர்) பலப்படுத்திக்கொள்ளுங்கள். அல்லாஹ்வுக்கு அஞ்சிக்கொள்ளுங்கள். (இம்மையிலும், மறுமையிலும்) நீங்கள் வெற்றியடைவீர்கள்!" (3: 200) எனும் வசனம் குறிப்பிடுகிறது.

அதேபோல் “(பயபக்தியுடையோர் எத்தகையோர் என்றால்,) அவர்கள் இன்பமான (செல்வ) நிலையிலும், துன்பமான (ஏழ்மை) நிலையிலும் (இறைவனின் பாதையில்) செலவிடுவார்கள். தவிர கோபத்தை அடக்கிக் கொள்வார்கள். மனிதர்(கள் செய்யும் பிழை)களை மன்னிப்போராய் இருப்பார்கள். (இவ்வாறு அழகாக) நன்மை செய்வோரையே அல்லாஹ் நேசிக்கின்றான்" (3: 134) எனும் வசனம் மனித உணர்ச்சிகளைக் கட்டுப்படுத்த வேண்டிய அவசியத்தை வலியுறுத்துகின்றது.

\section{VIII. சேவைநாடியின் நடத்தையை சீர்படுத்தல்}

அல்குர்ஆன் மற்றும் அஸ்ஸுன்னாவின் அடிப்படையிலமைந்த ஆற்றுப்படுத்தல் செயன்முறையின் இலக்குகளுள் சேவைநாடியின் நடத்தையை சீர்படுத்துவதும் ஒன்றறகும் [17].

எடுத்துக்காட்டாக "நன்மையும் தீமையும் சமமாக மாட்டா. நீங்கள் (தீமையை) நன்மைமைக் கொண்டே தடுத்துக் கொள்வீராக! அப்பொழுது, யாருக்கும் உமக்குமிடையே, பகைமை இருந்ததோ, அவர் உற்ற நண்பர் போல் ஆகிவிடுவார்" (41: 34) எனும் வசனம் இக்கருத்தைத் தெளிவுபடுத்துவதை அவதானிக்கலாம்.

\section{4. அல்குர்ஆன் மற்றும் அஸ்ஸூன்வை அடிப்படையாகக் கொண்ட ஆற்றுப்படுத்தலின் படிமுறைகள்}

அல்குர்ஆன் மற்றும் அஸ்ஸுன்வை அடிப்படையாகக் கொண்ட ஆற்றுப்படுத்தல் செயற்பாடு பல்வேறு படிமுறைகளைக் கொண்டதாகும் [13].

\section{I. தவறை/ குற்றத்தை ஒப்புக் கொள்ளல்}

அல்குர்ஆன் மற்றும் அஸ்ஸுன்னாவை அடிப்படையாகக் கொண்ட ஆற்றுப்படுத்தல் செயன்முறையின் முதல் படிமுறை தான் இழைத்த குற்றத்தை உணர்வதாகும். மனிதன் இழைக்கும் குற்றம் மற்றும் பாவத்தினால் அவனது உள்ளத்துக்குள் ஏற்படும் குற்ற உணர்விலிருந்து அவனை விடுவிப்பதற்காகவே இச்செயன்முறை மேற்கொள்ளப்படுகிறது. இதனூடாக மனித உள்ளத்துக்குள் ஏற்படும் விரக்தி மற்றும் விரிசல் நிலையிலிருந்து அவனை விடுவித்து உள அமைதியைத் தோற்றுவிக்க முடியும் [18].

\section{II. பாவமன்னிப்பு}

ஒரு மனிதன் குற்றத்தை உணர்வதனூடாக மாத்திரம் உளப்பிரச்சினைகளிலிருந்து அவனால் மீண்டுவிட முடியாது என்ற வகையில் அவன் தான் இழைத்த குற்றங்களில் இருந்து மீள்வது மிகவும் அவசியமான ஒன்றாகும். இதனூடாகவே மனித உள்ளம் தூய்மையடைகிறது என்பதையே இஸ்லாமிய மூல ஆவணங்கள் வலியுறுத்துகின்றன. [19].

எடுத்துகாட்டாக “என் அடியார்களே! (உங்களில்) எவரும் வரம்பு மீறி தமக்குத்தாமே தீங்கிழைத்துக் கொண்ட போதிலும், அல்லாஹ்வுடைய ரஹ்மத்தில் அவர் நம்பிக்கையிழக்க வேண்டாம். நிச்சயமாக அல்லாஹ் பாவங்கள் யாவற்றையும் மன்னிப்பான். நிச்சயமாக அவன் மிக்க மன்னிப்பவன். மிக்க கருணையுடையவன்" (என்று நான் கூறியதை நபியே!) நீர் கூறுவீராக" (39: 53) எனும் அல்குர்ஆனிய வசனம் ஒரு மனிதன் எவ்வகையான குற்றங்களை இழைத்து மன உளைச்சலுக்கு உட்படினும், பூரண இறை நம்பிக்கையுடன் பாவமீட்சியில் ஈடுபடுவதனூடாக உள அமைதியைப் பெற்றுக் கொள்ள முடியும் எனும் கருத்தை வலியுறுத்துவதை அவதானிக்க முடிகிறது. 


\section{III. சமூக மரபுகளுக்கு இயைபான புதிய ஆளுமை உருவாக்கம்}

தனது சீரற்ற சிந்தனைகள் மற்றும் செயற்பாடுகளின் விளைவாக பல்வேறு உளவியல் சவால்களுக்கு முகங்கொடுக்கும் மனிதன் தன்னைச் சூழவுள்ள சமூகத்தின் மூலம் தான் புறமொதுக்கத்துக்கு

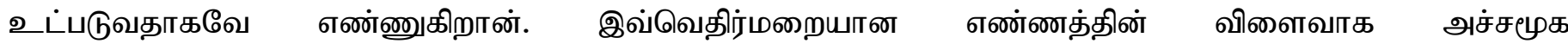
அங்கத்தவர்களுடன் முறையாக இடைவினை புரிய முடியாத நிலை அவனுக்குள் உருவாகிறது. இந்நிலையைத் தவிர்க்க உள அமைதி, தன்னம்பிக்கை, சுயமரியாதை மற்றும் பிறரை கண்ணியப்படுத்தல் போன்ற நேர்மறையான எண்ணங்களை வளர்ப்பது அவசியமானதாகும். இதனூடாக சீரிய சிந்தனை மற்றும் சாதகமான மனப்பான்யை மனிதனுக்குள் தோற்றுவிக்க முடியும் [20].

இதனையே “மக்களுடன் கலந்து வாழ்ந்து அவர்களின் மூலம் ஏற்படும் தீங்குகளை சகித்துக் கொள்ளும் இறை விசுவாசி மக்களுடன் கலந்து வாழாத, அவர்களால் ஏற்படும் தீங்குகளை சகித்துக் கொள்ளாத இறை விசுவாசியை விட சிறந்தவனாவான்" (இப்னு மாஜா, 3273) எனும் நபிமொழி உணர்த்துகிறது.

\section{IV. சேவைநாடியிடம் நேர்மறையான சிந்தனைகள் மற்றும் விழுமியங்களை உருவாக்கல்}

சிகிச்சையளிக்கும் போது சுயமாக சேவைநாடியின் நடத்தை மாற்றங்களை ஏற்படுத்த முடியும் என்ற நிலையை ஆற்றுப்படுத்துனர் உணரும் போது சேவைநாடியின் உள்ளத்தில் நேர்மறையான மாற்றங்களை ஏற்படுத்துவது அவசியமாகும். அத்துடன் அவரது நடத்தையில் சீரிய விழுமியங்கள் பிரதிபலிக்கும் வகையில் மாற்றங்களை ஏற்படுத்துவதும் ஆற்றுப்படுத்துனரின் கடமையாகும் [13].

மேற்கூறப்பட்டவையே அல்குர்ஆன் மற்றும் அஸ்ஸுன்வை அடிப்படையாகக் கொண்ட ஆற்றுப்படுத்தலின் படிமுறைகளாகும்.

\section{5. ஆற்றுப்படுத்தல் வழிமுறைகள் தொடர்பான ஸுன்னாவின் நடைமுறை வழிகாட்டல்கள்}

உளவளத்துணைச் செயற்பாட்டை மேற்கொள்ளும் போது ஓர் உளவளத்துணையாளர் பிரயோகிக்க முடியுமான பல்வேறு வழிமுறைகள் நபி (ஸல்) அவர்கள் மூலம் பிரயோகிக்கப்பட்டுள்ளன [21]. அவற்றுள் சில பின்வருமாறு:

\section{I. உரையாடல் முறை}

மனித சிந்தனைகளில் ஏற்படும் தவறுகள் தவிர்க்கப்பட்டு சிந்தனைத் தெளிவு ஏற்பட இம்முறை பெரிதும் துணை புரிகிறது. மனிதனின் உளவியல் பிரச்சினைகள் அனைத்துக்கும் தர்க்க ரீதியான தீர்வுகள் பெறுவதற்கு இம்முறை அவசியமானதாகும் [13]. நபி (ஸல்) அவர்கள் பல்வேறு சந்தர்ப்பங்களில் இம்முறையைப் பயன்படுத்தியுள்ளார்கள். எடுத்துகாட்டாக பின்வரும் இரு நிகழ்களைக் குறிப்பிட முடியும்.

\section{i. அன்ஸாரிகளுடனான நபி (ஸல்) அவர்களின் உரையாடல்}

ஹனனைன் யுத்தம் நடைபெற்றதன் பிற்பாடு அந்த யுத்தத்தில் கிடைக்கப் பெற்ற (கனீமா) பொருட்களை நபி (ஸல்) அவர்கள் குரைஷ் கோத்திரத்தையும் ஏனைய சில அரபுக் கோத்திரங்களையும் சார்ந்த புதிதாக இஸ்லாத்தை ஏற்றுக் கொண்ட மக்களிடையே பகிர்ந்தளித்தார்கள். எனினும் மதீனாவைச் சார்ந்த அன்ஸாரிகளுக்கு எப்பங்கும் வழங்கப்படவில்லை. அப்போது அவர்களில் ஒரு சாரார் இவ்விடயம் தொடர்பில் அதிருப்தியுற்று பல்வேறு குறைகளை தங்களுக்குள் பேசிக் கொண்டனர். அவர்களில் ஒருவர் “நபி (ஸல்) அவர்கள் தனது கோத்திரத்தாரை சந்தித்து விட்டார் (அவர்களுக்கு வேறு உதவியாளர்கள் கிடைத்துவிட்டார்கள் எனக் குறிப்பிட்டார்)". (இதனை அவதானித்த) ஸ. .த் பின் உபாதா (ரழி) அவர்கள் நபியவர்களிடம் வந்து “இறை தூதரே! யுத்தத்தில் கிடைக்கப்பெற்றவற்றை நீர் பகிர்ந்தளித்த விதம் தொடர்பில் சிலர் அதிருப்தியடைந்துள்ளனர். அவற்றை நீர் உமது கோத்திரத்தாருக்கே பகிந்தளித்து விட்டீர். 
ஏனைய அரேபியக் கோத்திரங்களுக்கும் பல்வேறு அன்பளிப்புக்களை வழங்கினீர். எனினும் அன்ஸாரிகளுக்கு ஒன்றும் கிடைக்கப்பெறவில்லை" எனக் குறிப்பிட்டார்கள். அப்போது நபி (ஸல்) அவர்கள் “ஸ.:தே! இவ்விடயம் தொடர்பில் நீர் என்ன கருதுகிறீர்” என வினவினார்கள். அப்போது "இறைதூதரே! நான் எனது கோத்திரத்தில் ஒருவர் தான். எனினும் நான் அவ்வாறு கூறவில்லை" என அவர் பதிலளித்தார். பின்னர் நபி (ஸல்) அவர்கள் அவர்களை ஒரு கூடாரத்தில் ஒன்று சேர்க்குமாறு அவரிடம் வேண்ட, அவரும் அவர்களை அக்கூடாரத்தில் ஒன்று சேர்த்தார். பின்னர் அங்கு சமுகமளித்த நபி (ஸல்) அவர்கள் அன்ஸாரிகளை நோக்கி “(எனது செயற்பாட்டில்) நீங்கள் (கவலையுற்று) அதிருப்தியுடன் இருப்பதாக எனக்கு அறியக்கிடைத்தது! நீங்கள் வழிதவறி இருந்தபோது இறைவன் உங்களுக்கு நேர்வழி காட்டவில்லையா? நீங்கள் ஏழ்மையில் இருந்த போது இறைவன் உங்களுக்கு வசதிகளை வழங்கவில்லையா? நீங்கள் (உங்களுக்கிடையே) விரோதத்துடன் இருந்த போது இறைவன் உங்களது உள்ளங்களுக்கிடையே பிணைப்பை ஏற்படுத்தவில்லையா? என வினவ அதற்கவர்கள் “ஆம்! அல்லாஹ்வின் தூதரே" என பதிலளித்தார்கள். அப்போது “அன்ஸாரிகளே! நீங்கள் பதிலளிக்க மாட்டீர்களா?" என வினவினார்கள். அதற்கு “இறைதூதரே நாங்கள் எவ்வாறு பதிலளிக்க வேண்டும்?" என வினவினார்கள். அப்போது நபி (ஸல்) அவர்கள், “இறைவன் மீது ஆணையாக! நீங்கள் நாடியிருப்பின் (என்னிடம்) நீர் (உமது சமூகத்தால்) பொய்ப்பிக்கப்பட்டு வந்தபோது நாமே உம்மை உண்மைப்படுத்தினோம்; நீர் மோசடிக்கு உட்படுத்தப்பட்ட போது நாமே உமக்கு உதவினோம்; நீர் விரட்டப்பட்ட போது நாமே உமக்கு அடைக்களமளித்தோம்; நீர் ஆதரவற்றவராக இருந்தபோது நாமே உமக்கு ஆதரவளித்தோம்" எனக் கூறியிருக்க முடியும் என பதிலளித்தார்கள். (பின்னர்) அன்ஸாரிகளே! அற்ப உலகப் பொருட்களுக்ாக நீங்கள் இவ்வாறு அதிருப்தியுற வேண்டுமா!? என வினவி, பின்னர் “அன்ஸாரிகளே! (ஏனைய) மக்கள் ஆடு, ஒட்டகம் போன்றவற்றைக் கொண்டு செல்ல நீங்கள் இறைவனின் தூதரை (உங்களுடன்) கூட்டிச் செல்வதை பொருந்திக்கொள்ள மாட்டீரா? என வினவினார்கள். பின்னர் “இறைவன் மீது ஆணையாக! ஹிஜ்ரத் மட்டும் இல்லையெனில் நானும் அன்ஸாரிகளுள் ஒருவராகவே இருந்திருப்பேன்; மக்கள் அனைவரும் ஒரு பள்ளத்தாக்கில் நுழைந்து அ அன்ஸாரிகள் வேறு ஒ ஒரு பள்ளத்தாக்கில் நுழைவார்களாயின் நான் அன்ஸாரிகள் நுழைந்த பள்ளாத்தாக்கிலேயே நுழைந்திருப்பேன்" எனக் குறிப்பிட்டு பின்னர் “இறைவா! அன்ஸாரிகளுக்கு அருள் புரிவாயாக! அவர்களின் பிள்ளைகளுக்கு அருள் புரிவாயாக! அவர்களின் பிள்ளைகளின் பிள்ளைகளுக்கு அருள் புரிவாயாக!" எனப் பிரார்த்தித்தார்கள். அப்போது அங்கிருந்த அனைவரும் தங்களது தாடைகள் நனையுமளவு தேம்பித் தேம்பி அழுது “அல்லாஹ்வின் தூதரையே எமக்கான பங்காக நாம் பொருந்திக் கொள்கிறோம்" எனக் கூறினார்கள். பின்னர் அங்கிருந்து நபி (ஸல்) அவர்கள் சென்றவுடன் அன்ஸாரிகளும் கலைந்து சென்று விட்டனர் (அஹ்மத், 11730).

மேற்கூறப்பட்ட நிகழ்வினுடாக அங்கு ஏற்பட்ட பிணக்கின் போது உரையாடல் முறையைப் பயன்படுத்தி, அன்ஸாரிகளுக்குத் தேவையான வழிகாட்டல்களை வழங்கி, அப்பிணக்கை வெற்றிகரமாகத் தீர்த்து வைத்ததோடு அவர்களின் மனதில் நேர்மறையான உணர்வுகளை நபி (ஸல்) ஏற்படுத்தியுள்ளமை தெளிவாகிறது.

\section{ii. விபசாரத்தில் ஈடுபடுவதற்கு அனுமதி வழங்கும் படி வினவிய வாலிபருடனான நபி (ஸல்) அவர்களின் உரையாடல்.}

“ஒரு வாலிபர் நபி (ஸல்) அவர்களிடம் வந்து இறைதூதரே! எனக்கு விபசாரத்தில் ஈடுபட அனுமதியளியுங்கள் என வினவினார். அப்போது அங்கிருந்தவர்கள் அவரை விரட்ட முயன்றனர். (எனினும்) நபி (ஸல்) அவர்கள் அவரை அருகில் அழைக்க, அவரும் (அருகில்) வந்து அமர்ந்து கொண்டார். அப்போது அவரிடம் அதனை உனது தாய்க்கு விரும்புவீரா? (உனது தாய் வேறு ஒருவருடன் விபசாரத்தில் ஈடுபடுவதை) விரும்புவீரா? 
என வினவ அதற்கவர் “மாட்டேன்” என பதிலளித்தார். பின்னர் (நபி) (ஸல்) அவர்கள் “எவரும் தனது தாய் அவ்வாறு ஈடுபடுவதை விரும்பமாட்டார்" எனக் கூறினார்கள். பின்னர் அவரிடம் உமது மகளுக்கு அதனை நீர் விரும்புவீரா" என வினவ, அவர் “மாட்டேன்" என பதிலளித்தார். அப்போது (நபி) (ஸல்) அவர்கள் “எவரும் தனது மகளுக்கு அதனை விரும்பமாட்டார்" எனக் குறிப்பிட்டார்கள். பின்னர் அவரிடம் “உனது சகோதரிக்கு அதனை நீர் விரும்புவீரா?" எனக் கேட்க, அவர் “மாட்டேன்" என பதிலளித்தார். அப்போது (நபி) (ஸல்) அவர்கள் “எவரும் தனது சகோதரிக்கு அதனை விரும்பமாட்டார்" எனக் குறிப்பிட்டார்கள். பின்னர் “உனது தந்தையின் சகோதரிக்கு (மாமி) அதனை நீர் விரும்புவீரா?" எனக் கேட்க, அவர் “மாட்டேன்” என பதிலளித்தார். அப்போது (நபி) (ஸல்) அவர்கள் “எவரும் தனது தந்தையின் சகோதரிக்கு அதனை விரும்பமாட்டார்" எனக் குறிப்பிட்டார்கள். பின்னர் “உனது தாயின் சகோதரிக்கு (சின்னம்மா/ பெரியம்மா) அதனை நீர் விரும்புவீரா?" எனக் கேட்க, அவர் “மாட்டேன்" என பதிலளித்தார். அப்போது (நபி) (ஸல்) அவர்கள் “எவரும் தனது தாயின் சகோதரிக்கு அதனை விரும்பமாட்டார்" எனக் குறிப்பிட்டார்கள். பின்னர் அவரின் மேல் தனது கையை வைத்து "இறவைா! இவரின் பாவத்தை மன்னித்து, உள்ளத்தைத் தாய்மைப்படுத்தி, இவரது அபத்தை பாதுகாப்பாயாக!” எனப் பிரார்த்தித்தார்கள். அதன் பின்னர் அவ்வாலிபன் அதன் பக்கம் திரும்பிப் பார்க்கவே இல்லை (அஹ்மத், 22211).

மேற்கூறப்பட்ட சினேகபூர்வ உரையாடல் முறையினூடாக அவ்வாலிபனின் பிறழ்வு நடத்தையை நபி (ஸல்) அவர்கள் சாமர்த்தியமாகக் கையாண்டு வெற்றிகரமாக அவரது நடத்தையில் சாதகமான மாற்றங்களை ஏற்்படுத்தியிருப்பது தெளிவாகிறது.

\section{II. உதாரணங்களைக் குறிப்பிட்டு தெளிவுபடுத்தும் முறை}

ஒரு விடயம் குறித்த தெளிவை வழங்குவதற்காக உதாரணங்களைக் குறிப்பிடும் முறை ஹதீஸ்களில் கையாளப்பட்டுள்ளது. (பெறுமதியான அல்லது பெறுமதியற்ற) ஒரு அறிமுகமான விடயத்தை அறிமுகமற்ற ஒரு விடயத்துடன் ஒப்பிட்டு தேவையான விடயத்தைத் தெளிவுபடுத்துவதே இம்முறையின் பிரதான நோக்கமாகும் [13]. எடுத்துகாட்டாக பின்வரும் விடயங்களைக் குறிப்பிட முடியும்.

\section{i. நல்ல மற்றும் தீய நண்பர்களுக்கான உதாரணம்}

நல்ல நண்பனுக்கும் தீய நண்பனுக்கும் உதாரணம் கஸ்தூரி வைத்திருப்பவரும் கொல்லனின் உலையுமாகும்! கஸ்தூரி வைத்திருப்பவரிடமிருந்து நீர் அதை விலைக்கு வாங்கலாம் அல்லது அதன் நறுமணத்தையாவது பெறலாம்! கொல்லனின் உலை உம்முடைய வீட்டையோ உம்முடைய ஆடையையோ எரித்து விடும் அல்லது அவனிடமிருந்து கெட்ட வாடையை நீர் பெற்றுக் கொள்வீர்! (புகாரி, 2101).

நல்ல நண்பரைத் தேர்ந்தெடுத்துக் கொள்வதன் முக்கியத்துவத்தை மேற்கூறப்பட்ட நபிமொழியினூடாக நபி (ஸல்) அவர்கள் குறிப்பிட்டுள்ளார்கள். நவீன உளவளத்துணை அறிஞர்கள் ஒரு தனிமனிதரின் நடத்தைகள் மற்றும் சிந்தனைகளில் அவரது நண்பர்கள் நேர்மறையான அல்லது எதிர்மறையான தாக்கத்தை செலுத்துவதை தமது ஆய்வுகளினூடாக உறுதிப்படுத்தியுள்ளனர் [13]. அக்கருத்தையே நபி (ஸல்) மேற்கூறப்பட்ட உதாரணத்தினூடாகத் தெளிவுபடுத்தி, தீய நட்பு பல்வேறு இழப்புக்களையும் சிக்கல்களையும் தோற்றுவிக்கும் என்பதை வலியுறுத்தியுள்ளார்கள்.

\section{ii. இறைவனின் வரம்புகளுக்கு கட்டுப்படுபவர் மற்றும் அதனை மீறுபவருக்கான உதாரணம்}

அல்லாஹ்வின் (சட்ட) வரம்புகளுக்குக் கட்டுப்பட்டு நடப்பவனுக்கும் அவற்றை மீறி நடப்பவனுக்கும் உவமை ஒரு சமுதாயத்தைப் போன்றதாகும். அவர்கள் கப்பலில் (தங்களுக்கு இடம் பிடிப்பதற்காகச்) சீட்டுக் குலுக்கிப் போட்டார்கள். (அதன்படி) அவர்களில் சிலருக்குக் கப்பலின் மேல் தளமும் சிலருக்குக் கீழ்த் தளமும் கிடைத்தது. 
கீழ்த் தளத்தில் இருந்தவர்களுக்குத் தண்ணீர் தேவைப்பட்டபோது (அதைக் கொண்டு வர) அவர்கள் மேல் தளத்தில் இருப்பவர்களைக் கடந்து செல்ல வேண்டியிருந்தது. (அதனால் மேலே இருந்தவர்களுக்குச் சிரமம் ஏற்பட்டது). அப்போது, கீழ்த் தளத்தில் இருந்தவர்கள் (தமக்குள்) நாம் (தண்ணீருக்காக) நம்முடைய பங்கில் (கீழ்த் தளத்தில்) ஓட்டையிட்டுக் கொள்வோம். நமக்கு மேலே இருப்பவர்களைத் தொந்தரவு செய்யாமலிருப்போம் என்று பேசிக் கொண்டார்கள். அவர்கள் விரும்பியபடி செய்து கொள்ள அவர்களை மேல் தளத்தில் உள்ளவர்கள் விட்டுவிட்டால் (கப்பலில் இ இருப்பவர்கள்) அனைவரும் அழிந்து போவார்கள். (ஓட்டையிட விடாமல்) அவர்களின் கரத்தைப் பிடித்துக் கொள்வார்களாயின் அவர்களும் தப்பிப் பிழைத்துக் கொள்வார்கள். (அவர்களுடன் மற்ற) அனைவரும் தப்பிப் பிழைத்துக் கொள்வார்கள் (புகாரி, 2493).

மேற்கூறப்பட்ட ஹதீஸில் உள அமைதி மற்றும் உள நிம்மதி போன்றவற்றுக்கான முக்கிய அடிப்படைகளுள் ஒன்றை நபி (ஸல்) அவர்கள் குறிப்பிட்டார்கள். (சமூக) கூட்டுப் பொறுப்பு எனும் விடயமே அவ்வடிப்படையாகும். ஒரு சமூகத்தின் பாதுகாப்பு மற்றும் அமைதி ஆகியவை அச்சமூக அங்கத்தவர்கள் அனைவரினதும் பொறுப்பாகும். அதனை முறையாக நிறைவேற்றத் தவறும் பட்சத்தில் அது அனைவருக்கும் அச்சுறுத்தலான விடயமாகும் எனும் கருத்தையே மேற்கூறப்பட்ட நபி மொழி வலியுறுத்துகிறது.

\section{iii. இறைவனிடம் இவ்வுலகுக்கான பெறுமானத்தை தெளிவுபடுத்தும்உதாரணம்}

ஒருமுறை நபி (ஸல்) அவர்கள் உயரமான இடத்தில் இருந்த ஒரு சந்தையில் நடந்து சென்றார்கள். அவரது இரு புறமும் மக்கள் இருந்தனர். அப்போது காதற்ற இறந்து கிடந்த ஒரு ஆட்டுக் குட்டியைக் கடந்து சென்ற அவர்கள், அதனைக் குறிப்பிட்டு "உங்களில் எவரேனும் இதனை ஒரு திர்ஹம் பணத்துக்கு வாங்கிக் கொள்ள விரும்புவீரா?" என வினவ, அங்கிருந்தவர்கள் “அது எவ்வித பயனுமற்றது. அதனை (வைத்து) ஒன்றும் செய்ய முடியாது. (எனவே) எங்களில் எவரும் அதனை விரும்ப மாட்டார்" என பதிலளித்தனர். அப்போது நபி (ஸல்) அவர்கள் அது இலவசமாகக் கிடைப்பதை உங்களில் எவரும் விரும்புகிறீரா?" எனக் கேட்க, அதற்கவர்கள் “காதுகளற்ற ஆட்டுக் குட்டியாக இருப்பதனால் அது உயிருடன் இருக்கும் போதே எங்களில் எவரும் அதனை விரும்பமாட்டார். அவ்வாறு இருக்கையில் அப்பிராணி இறந்து கிடக்கும் போது எவ்வாறு? (அதனை விரும்புவர்?)" எனக் கேட்டார்கள். அதற்கு நபி (ஸல்) அவர்கள் “இறைவன் மீது ஆணையாக! இப்பிராணி உங்களுக்கு இழிவானதாக இருப்பதை விட இவ்வுலகம் இறைவனிடத்தில் பெறுமதியற்றது" எனக் குறிப்பிட்டார்கள் (முஸ்லிம், 2957).

கட்டற்ற எதிர்பார்ப்புகள் மற்றும் பொருள் மோகம் என்பவையே பலரின் உளவியல் ரீதியான பிரச்சினைகளுக்கான மூலக் காரணிகளாகும். எனவே, காதற்ற இறந்த ஆட்டுக் குட்டியை உதாரணமாகக் குறிப்பிட்டு நபி (ஸல்) அவர்கள் இவ்வுலகின் உண்மையான பெறுமானத்தைத் தெளிவுபடுத்தினார்கள்.

\section{III. செயல்களினூடாகத் தெளிவுபடுத்தும் முறை}

ஒரு விடயம் தொடர்பில் செயல்களினூடாகத் தெளிவுபடுத்தும் முறை உளவளத்துணைச் செயற்பாட்டில் முக்கிய வழிமுறைகளுள் ஒன்றாகக் கருதப்படுகிறது. அறிவியல்சார் விடயங்களும் மனித உள்ளங்களில் பண்பாடுகள் மற்றும் பெறுமானங்கள் தொடர்பான புரிதலும் இவ்வழிமுறையினூடாகவே தெளிவுபடுத்தப்படுகிறது [18]. இம்முறை பல்வேறு நபிமொழிகளில் பயன்படுத்தப்பட்டுள்ளது. எடுத்துக்காட்டாக பின்வருவனவற்றைக் குறிப்பிட முடியும்.

\section{i. தொழும் முறை}

நபி (ஸல்) அவர்கள் பள்ளிக்கு வந்தார்கள். ஒரு மனிதரும் (அந்த நேரத்தில்) பள்ளிக்கு வந்து தொழலானார். (தொழுது முடித்ததும்) நபி (ஸல்) அவர்களுக்கு அவர் ஸலாம் கூறினார். நபி (ஸல்) அவர்கள் பதில் ஸலாம் கூறினார்கள். பின்னர் நபி (ஸல்) அவரிடம் “நீர் திரும்பச் ென்று தொழுவீராக, (ஏனெனில்) நீர் தொழவே இல்லை” எனக் கூற, அந்த மனிதர் முன்பு தொழுதது போன்றே மீண்டும் தொழுதுவிட்டு வந்து, 
நபி (ஸல்) அவர்களுக்கு ஸலாம் கூறினார். “திரும்பவும் தொழுவீராக! நீர் தொழவே இல்லை" என மீண்டும் நபி (ஸல்) அவர்கள் கூறினார்கள். இவ்வாறு மூன்று முறை நடந்தது. அதன் பிறகு அந்த மனிதர் சத்திய மார்க்கத்துடன் உங்களை அனுப்பியுள்ள இறைவன் மீது ஆணையாக! இவ்வாறு தொழுவதைத் தவிர வேறு எதையும் நான் அறிந்திருக்கவில்லை. எனவே, எனக்குக் கற்றுத் தாருங்கள்! என்று கேட்டார். நீர் தொழுகைக்காக நின்றதும் தக்பீர் கூறும்! பின்னர் அல்குர்ஆனில் உமக்குத் தெரிந்தவற்றை ஓதும்! பின்னர் அமைதியாக ருகூவு செய்வீராக! பின்னர் ருகூவிலிருந்து எழுந்து சரியான நிலைக்கு வருவீராக! பின்னர் நிதானமாக ஸஜ்தா செய்வீராக! ஸஜ்தாவிலிருந்து எழுந்து நிதானமாக உட்கார்வீராக! இவ்வாறே உம்முடைய எல்லாத் தொழுகையிலும் செய்து வருவீராக! என நபி (ஸல்) அவர்கள் கூறினார்கள் (புகாரி, 6251).

சில மனிதர்கள் ஸஹ்ல் இப்னு ஸஅத் (ரலி) அவர்களிடம் வந்து நபி (ஸல்) அவர்களின் மிம்பர் எதனால் தயாரிக்கப்பட்டுள்ளது என்பது பற்றிக் கேட்டனர். அதற்கு ஸஹ்ல் (ரலி) “இறைவன் மீது ஆணையாக அது எதனால் தயாரிக்கப்பட்டுள்ளது என்பதை நான் நன்கறிவேன். அது வைக்கப்பட்டு, அதன் மீது நபி (ஸல்) அமர்ந்த முதல் நாளிலேயே அதனை நான் கண்டிருக்கிறேன்". நபி (ஸல்) அவர்கள் ஒரு பெண்மணியிடம் அப்பெண்மணியின் பெயரை ஸஹ்ல் (ரழி) கூறினார். தச்சு வேலை செய்யும் உன்னுடைய பணியாளரிடம் “நான் மக்களுக்கு உரையாற்றும்போது அமர்ந்து கொள்வதற்கேற்ற மேடையைச் செய்து தரச் சொல்” என்று சொல்லி அனுப்பினார்கள். அப்பெண் தம் பணியாளரிடம் இதைக் கூறினார். அப்பணியாளார் $\therefore$ காபா எனும் பகுதியில் உள்ள மரத்தில் அதைச் செய்து வந்தார். அப்பெண் அதை நபி (ஸல்) அவர்களிடம் கொடுத்தனுப்பினார். அதை உரிய இடத்தில் வைக்குமாறு நபி (ஸல்) அவர்கள் கட்டளையிட்டார்கள். பின்னர் அதன் மீது ஏறி தக்பீர் சொல்லித் தொழுதார்கள். பின்னர் அதன் மீதே ருகூஉம் செய்தார்கள். பின்னர் மிம்பரிலிருந்து இறங்கி அது வைக்கப்பட்ட இடத்துக்கு அருகில் ஸஜ்தா செய்தார்கள். பின்னர் அவ்வாறே மீண்டு வந்து தொழுகையை நிறைவேற்றினார்கள். பின்னர் “மக்களே நீங்கள் என்னைப் பின்பற்றித் தொழுவதற்காகவும் எனது தொழுகையை அறிந்து கொள்வதற்காகவுமே இவ்வாறு செய்தேன்” எனக் கூறினார்கள் (புகாரி, 2094).

மேற்கூறப்பட்ட இரு ஹதீஸ்களில் உளவளத்துணையின் இரு முக்கிய நுட்பங்கள் தொடர்பில் தெளிவுபடுத்தப்பட்டுள்ளது. முதலாவது ஹதீஸில் ஒரு மனிதன் தனது தவறுகளை ஏற்றுக் கொள்வது தொடர்பில் நபி (ஸல்) அவர்கள் தெளிவுபடுத்தியுள்ளார்கள். சேவைநாடி தனது தவறுகளை ஏற்றுக் கொள்வது உளவளத்துணையின் முக்கிய அடிப்படைகளுள் ஒன்றாகும். தவறுகளை ஏற்றுக் கொண்ட பின்னர் அவருக்கு வழங்கப்பட வேண்டிய ஆலோசனைகள் வழங்கப்பட வேண்டும். சரியான முறையில் எவ்வாறு தொழுகையை அமைத்துக் கொள்ள வேண்டும் என்பது தொடர்பில் நபி (ஸல்) அவர்கள் மிம்பரில் இருந்து தெளிவுபடுத்தியமையானது தவறுகளை ஒர்புக் கொண்ட பின்னர் அவருக்குத் தேவையான ஆலோசனைகள் வழங்கப்படுவதற்கான எடுத்துக்காடாக அமையப்பெற்றுள்ளது.

\section{ii. மீள்பரிசீலனை}

“நீ உன்னுடைய படுக்கைக்குச் செல்லும்போது தொழுகைக்குச் செய்வது போல் வுழூச் செய்து கொள். பின்னர் உன்னுடைய வலக்கைப் பக்கமாகச் சாய்ந்து படுத்துக் கொள். பின்னர் “யா அல்லாஹ்! நான் என்னுடைய முகத்தை உன்னிடம் ஒப்படைத்தேன். என்னுடைய காரியங்களை உன்னிடம் விட்டுவிட்டேன். என்னுடைய முதுகை உன் பக்கம் சாய்த்து விட்டேன். உன்னிடத்தில் ஆதரவு வைத்தவனாகவும் உன்னைப் பயந்தவனாகவும் இதைச் செய்கிறேன். உன்னை விட்டுத் தப்பிச் செல்லவும் உன்னை விட்டு ஒதுங்கி விடவும் உன் பக்கமே தவிர வேறிடம் இல்லை. யா அல்லாஹ்! நீ இறக்கிய உன்னுடைய வேதத்தை நான் நம்பினேன். நீ அனுப்பிய உன்னுடைய நபியையும் நம்பினேன்" என்ற பிரார்த்தனைய நீ செய்து கொள். 
(இவ்வாறு நீ சொல்லிவிட்டு உறங்கினால்) அந்த இரவில் நீ இறந்துவிட்டால் நீ தாய்மையானவனாய் ஆகிவிடுகிறாய். இந்தப்பிரார்த்தனையை உன்னுடைய (இரவின்) கடைசிப் பேச்சாக ஆக்கிக் கொள்" என்று என்னிடம் இறைத்தூதர் (ஸல்) கூறினார்கள். நான் நபி (ஸல்) அவர்களிடம் இந்தப் பிரார்த்தனையைத் திரும்ப ஓதிக் காண்பித்தேன். அப்போது “நீ அனுப்பிய உன்னுடைய நபியையும் நம்பினேன் என்பதற்குப் பதிலாக உன்னுடைய ரஸுலையும் நம்பினேன் என்று சொன்னேன். உடனே நபி (ஸல்) அவர்கள் “இல்லை, நீ அனுப்பிய உன்னுடைய நபியை நம்பினேன் என்று சொல்லும்" என எனக்குத் திருத்திக் கொடுத்தார்கள்““ என பர்ரா. இ இப்னு ஆஸிப் (ரலி) அறிவித்தார் (புகாரி, 247).

இதற்கு முன்னர் குறிப்பிடப்பட்ட நபிமொழியில் செயன்முறைகளின் ஊடாகத் தெளிவுபடுத்தும் வழிமுறை பன்படுத்தப்பட்ட அதேவேளை இந்நபிமொழியில் தவறுகளைத் திருத்தும் வழிமுறை பயன்படுத்தப்பட்டுள்ளது. ஒருவர் தனக்குள் தவறுகள் எவையேனும் ஏற்படும்போது அச்செயலை மீள்பரிசீலனைக்கு உட்படுத்தி அவற்றை திருத்திக் கொள்ளும் வழிமுறையையே நபி (ஸல்) அவர்கள் இந்நபிமொழியில் தெளிவுபடுத்தியுள்ளார்கள்.

\section{IV. அழகிய உபதேசங்கள் ஊடாகத் தெளிவுபடுத்தும் முறை}

ஒரு மனிதனுக்கு நலவை நாடும் நோக்கில் அவனுக்கு வழங்கப்படும் பிரத்தியேகமான அனைத்து வழிகாட்டல்களும் உபதேசங்களாகும். ஒரு மனிதனை தவறுகளில் இருந்து விடுவித்து நல்ல விடயங்கள் தொடர்பில் அவனைத் தெளிவூட்டுவதே இதன் பிரதான இலக்காகும். உளவளத்துணை ஆலோசகருக்கு ஆற்றுப்படுத்தல் செயன்முறையின் போது இம்முறை மிகவும் பயனுள்ளதாகும். இம்முறைக்கான எடுத்துக்காட்டுகளாக பின்வருவனவற்றைக் குறிப்பிட முடியும்.

\section{i. மார்க்கம் என்பது (பிறருக்கு) நலவை நாடுவதாகும்.}

“(இஸ்லாமிய) மார்க்கம் என்பது (பிறருக்கு) நலவு நாடுவதாகும். (அப்போது) அங்கிருந்தவர்கள் யாருக்கு நலவை நாடுவது (என வினவ, அதற்கு) இறைவனுக்கும், அவனது வேதத்துக்கும், அவனது தூதருக்கும், முஸ்லிம்களின் தலைவர்களுக்கும், பொது மக்களுக்கும் என நபி (ஸல்) அவர்கள் கூறினார்கள் (முஸ்லிம், 55).

மனிதர்களுக்கு மத்தியில் தேவையா நேரங்களில் தேவையான வழிகாட்டல்களை வழங்குவதன் ஊடாக அவர்களுக்கு நலவை நாடுவதன் அவசியம் தொடர்பில் இந்நபிமொழி விபரிக்கிறது.

\section{ii. இறைவன் மற்றும் இறைவிதி மீதான நம்பிக்கை}

இப்னு அப்பாஸ் (ரழி) அவர்கள் அறிவித்தார்.

“ஒருமுறை நான் (ஒட்டகத்தில்) நபியவர்களின் பின்னால் அமர்ந்து கொண்டேன். அப்போது நபியவர்கள் என்னிடம் “சிறுவனே உனக்கு சில விடயங்களை நான் கற்றுத் தருகிறேன்; நீ அல்லாஹ்வைப் பாதுகாத்துக் கொள். அவன் உன்னைப் பாதுகாப்பான். நீ அல்லாஹ்வைப் பாதுகாத்துக் கொள். அவனை உனக்கு முன்னால் நீ கண்டு கொள்வாய். நீ ஏதேனும் கேட்டால் இறைவனிடமே கேள். நீ உதவி தேடினால் இறைவனிடமே உதவி தேடு. அறிந்து கொள். மனித சமூகம் முழுவதும் ஒன்று சேர்ந்து உனக்கு ஏதேனும் ஒரு நன்மையை ஏற்படுத்த முயன்றாலும் இறைவன் விதித்தைத் தவிர (மேலதிகமாக) அவர்களால் உனக்கு நன்மை பயக்க முடியாது. அதேபோல் அவர்கள் அனைவரும் ஒன்றிணைந்து உனக்கு ஒரு கெடுதியை ஏற்படுத்த நாடினால் இறைவன் விதித்ததைத் தவிர (மேலதிகமாக) அவர்களால் உனக்கு கெடுதியிழைக்க முடியாது. பேனைகள் உயர்த்தப்பட்டுவிட்டன. ஏடுகள் காய்ந்து விட்டன" (திர்மிதி, 2516).

மேற்கூறப்பட்ட இரு நபிமொழிகளிலும் அழகிய உபதேசங்கள் ஊடாக தேவையான வழிகாட்டல்கள் வழங்கப்பட்டுள்ளமையை அவதானிக்க முடிகிறது. 


\section{6. உளவியல் பிரச்சினைகளை எதிர்கொள்வதற்கான அல்குர்ஆன் மற்றும் அஸ்ஸீன்னாவின் பொதுவான வழிகாட்டல்கள்}

\section{I. இறைவிதி}

இஸ்லாமிய உளவளத்துணையில் விதி (கழா, கத்ர்) பற்றிய நம்பிக்கை மிகப் பிரதானமானதாகும். இது பற்றி அல்குர்ஆன் “பூமியிலோ, அல்லது உங்களிலோ சம்பவிக்கிற எந்தச் சம்பவமும் - அதனை நாம் உண்டாக்குவதற்கு முன்னரே (லவ்ஹுல் மஹ்.:பூள்) ஏட்டில் இல்லாமலில்லை. நிச்சயமாக அது அல்லாஹ்வுக்கு மமக எளிதானதேயாகும்" (57: 22) என பிரஸ்தாபிக்கிறது

உள ரீதியாக எவ்வகையான நெருக்கடிகளுக்கு உட்படும்போதும் ஒவ்வொன்றும் இறைவனின் நாட்டப்படியே நடக்கிறது. அதைத்தவிர வேறெதுவும் நடைபெறப்போவதில்லை எனும் ஆழ்ந்த நம்பிக்கையானது உளவியல் ரீதியான மிகப்பெரும் பலத்தையும் எவ்வகையான உள நெருக்கடிகளையும் வெற்றிகரமாக எதிர்கொள்ளும் நிலையையும் மனிதனுக்குள் தோற்றுவிக்கிறது.

\section{II. பொறுமை}

உண்மையில் உளவியல் நோய்களை எதிர்கொள்வதற்கு பொறுமை மிக அவசியமாகும். வாழ்க்கையில் ஏற்படும் பிரச்சினைகள், சங்கடங்களின் போது துவண்டு விடாமல் இறைவன் மீது ஆழ்ந்த நம்பிக்கை கொண்டு அவற்றை எதிர்த்து போராடுவதும், பொறுமை காப்பதும் உளரீதியாக மனிதனை வலுப்படுத்தும் மிகச் சிறந்த வழி என்பதை “(நம்மைத் தேற்றும்) அல்லாஹ்வின் அருளில் இருந்து நம்பிக்கை இழக்காதீர்கள். ஏனென்றால் நிச்சயமாக காபிர்களின் கூட்டத்தைத் தவிர (வேறு யாரும்) அல்லாஹ்வின் அருளில் இருந்து நம்பிக்கை இழக்கமாட்டார்கள்" (28: 77) எனும் அல்குர்ஆனிய வசனம் உணர்த்துவதனூடாக மனிதனுக்கு ஏற்படும் உளவியல் நோய்களை வெற்றிகரமாக முறியடிப்பதற்கான உந்துதலை அவனுக்கு வழங்குகிறது.

\section{III. தற்கொலையின் பாரதாரம் தொடர்பான எச்சரிக்கை}

தற்கொலை தொடர்பான இஸ்லாமிய உளவளத்துணைக் கண்ணோட்டமானது வலுவானதும் மனித கண்ணியத்தை பேணக் கூடியதுமாகும். மனித உயிர், உள்ளம் மற்றும் ஆன்மா என்பன நெருங்கிய தொடர்புகளைக் கொண்டவையும் பாதுகாக்கப்பட வேண்டியவையும் என்பதால் உளவியல் நெருக்கடிகளால் தற்கொலைக்கு முயல்வதை இஸ்லாம் அனுமதிக்கவில்லை. அதை மிகப் பெரும் குற்றம் எனவும் கோழைத்தனம் எனவும் நரகில் நுழைவதற்கான காரணிகளில் அதுவும் ஒன்றாகும் எனவும் இஸ்லாம் குறிப்பிடுகிறது [22].

இதனைத் தெளிவுபடுத்தும் விதமாக நபி (ஸல்) அவர்கள் “கூறிய ஆயுதத்தால் தனது உயிரை மாய்த்துக் கொள்பவர் நரகில் நிரந்தரமாக தனது வயிற்றை கூறிய ஆயுதத்தால் குத்திக்கொள்வார், நஞ்சு குடித்து உயிரை மாய்த்துக்கொள்பவர் நரகிலும் நிரந்தரமாக நஞ்சையே குடிப்பார், மலையிலிருந்து கழே விழுந்து உயிரை மாய்த்துக்கொள்பவர் நரகிலும் நிரந்தரமாக அவ்வாறே விழுவார்" (புஹாரி, 5778) என்று குறிப்பிட்டுள்ளர்கள்.

உள நோய்களின் தீவிரத் தன்மையால் மனிதன் தனது அந்தஸ்தை மறந்து தற்கொலை செய்து கொள்வதை இஸ்லாம் வரவேற்கவே இல்லை. இதனாலே உலகை அச்சுறுத்தும் செயற்பாடுகளில் ஒன்றான தற்கொலை முஸ்லிம்களுக்கு ம மத்தியில் மிகக்குறைவாக காணப்படுகின்றது [23].

\section{IV. போதைப் பொருள் பாவனை தடைசெய்யப்பட்டுள்ளமை}

உளப்பிரச்சினைகளுக்கான மிக முக்கிய காரணம் போதைப்பொருள் பாவனையாகும். மனிதன் தனக்குத்தானோ அல்லது பிறருக்கோ தீங்கிழைப்பது இஸ்லாத்தில் முற்றாக தடை செய்யப்பட்டதாகும். இதனாலே போதையூட்டும் அனைத்தையும் அது தடை செய்கிறது. இது தொடர்பில் நபி (ஸல்) அவர்கள் “போதையூட்டும் அனைத்தும் தடை செய்யப்பட்டவையாகும்" (அபூ தாவூத், 3482) எனக் குறிப்பிட்டார்கள். இதனூடாக மனிதனின் உள நோய்களை கையாள்வதில் இஸ்லாம் எவ்வளவு அக்கறையுடன் செயற்படுகிறது என்பது தெளிவாகிறது. 


\section{7. உள நோய்கள் மற்றும் அவற்றுக்கான தீர்வுகள்}

உளப்பிரச்சினைகள் என அடையாளப்படுத்தப்படும் அனைத்துக்குமான நடைமுறைச் சாத்தியமான அழகிய தீர்வுகளை இஸ்லாம் முன்வைக்கிறது [9].

\section{I. மனச் சோர்வு (Depression)}

எடுத்துக்காட்டாக மனச்சோர்வு நோயானது மனித வாழ்வின் எதிர்மறை நிகழ்வுகளின் விளைவாகும். இந்நோயை தடுக்கும் முகமாக நேர்மறையான மனப்பாங்கினை வளர்த்துக் கொள்ளும் வழிகாட்டல்களை இஸ்லாம் வழங்குகிறது [22].

இது பற்றி அல்குர்ஆன் “நிச்சயமாக துன்பத்துடன் இன்பம் இருக்கிறது. எனவே, (வேலைகளிலிருந்து) நீர் ஓய்ந்ததும் (இறைவழியிலும், வணக்கத்திலும்) முயல்வீராக. மேலும், முழு மனத்துடன் உம் இறைவன் பால் சார்ந்து விடுவீராக" (94: 6-8) எனக் குறிப்பிடுகிறது.

\section{II. தவிப்பு (Anxiety)}

உளவியல் நோய்களுள் ஒன்றான பதகளிப்பு அல்லது தவிப்பு என்பது ஏதாவது ஒன்று நடப்பதை அக்கறையுடன் ஆவல் கொள்ளும் போதோ அல்லது நடக்கக்கூடாது என்று அச்சத்துடன் இருக்கும்போதோ மனதாலோ அல்லது உடலாலோ அடையும் தவிப்பு நிலையாகும். இது பயம், கவலை, நம்பிக்கையின்மை என்று பல உணர்வுகளை தோற்றுவிக்கும். பயம் என்பது தவிப்பை விட மேலோங்கிய உணர்வாகும், அது மனிதரை மொத்தமாக செயலிழக்க செய்ய முடியுமானதாகும் [22].

இறைவனுடனான தொடர்புகளை உறுதிப்படுத்தி தனது அனைத்துக் காரியங்களிலும் அவனை சார்ந்திருப்பதன் ஊடாக இவ்வுளவியல் நெருக்கடியிலிருந்து தன்னை விடுவிக்கலாம் என்பதாக இஸ்லாமிய உளவளத்துணை வழிகாட்டுகிறது. இது பற்றி அல்குர்ஆன் குறிப்பிடும் போது “ஒருபோதும் அல்லாஹ் விதித்ததைத் தவிர (வேறு ஒன்றும்) எங்களை அணுகாது. அவன் தான் எங்களுடைய பாதுகாவலன் என்று (நபியே!) நீர் கூறும்; மு.:மின்கள் அல்லாஹ்வின் மீதே பூரண நம்பிக்கை வைப்பார்களாக!" (28: 77) எனும் அல்குர்ஆனிய வசனமானது மனித மனதில் அதீத எதிர்பார்ப்பின் விளைவாகத் தோன்றும் தவிப்பு நிலையிலிருந்து தன்னை விடுவித்துக் கொள்வதற்கான தூண்டுதலை அவனுள் உண்டுபண்ணுகிறது.

\section{III. எண்ண சுழற்சி நோய் (Obsessive Compulsive Disorder - OCD)}

எண்ண சுழற்சி நோய் அல்லது மன சுழற்சி நோய் (Obsessive Compulsive Disorder - OCD) என்பது ஒரே வேலையை திரும்பத் திரும்பச் செய்யும் ஒரு வகை மனோநிலையாகும். உதாரணமாக கழிப்பறையை பயன்படுத்திய பின்னர் மீண்டும் மீண்டும் தண்ணீர் கொண்டு சுத்தம் செய்தல். இது பரவலாக பலரிடமும் அவதானிக்க முடியுமான ஒன்றாகும் [24].

இதனை இஸ்லாம் வஸ்வாஸ் என அடையாளப்படுத்தி இது ஷைத்தானின் தாண்டுதலால் மனித மனங்களில் தோன்றும் நிர்ப்பந்த நிலை என்பதையும் விளக்குகிறது. இதனையே அல்குர்ஆன் “எனினும் அவ்விருவருக்கும் மறைந்திருந்த அவர்களுடைய (உடலை) மானத்தை அவர்களுக்கு வெளிப்படுத்தும் பொருட்டு ஷைத்தான் அவ்விருவரின் உள்ளங்களில் (தவறான எண்ணங்களை) ஊசலாடச் செய்தான்" (07: 20) எனக் குறிப்பிடுகிறது.

இது ஷைத்தானின் செயற்பாடு என்பதால் இதனை விட்டும் இறைவனிடம் பாதுகாப்புத் தேடிக்கொள்ளுமாறு "பதுங்கியிருந்து வீண் சந்தேகங்களை உண்டாக்குபவனின் தீங்கை விட்டும் (இறைவனிடத்தில் நான் காவல் தேடுகிறேன்” (114: 4) எனும் வசனம் கற்றுத் தருகிறது. எனவே ஒரு செயலை செய்து முடித்த பின்னர் அது தொடர்பாக மனதில் நிலவும் தேவையற்ற திருப்தியின்மையால் அதனை மீண்டும் மீண்டும் செய்வதைத் தவிர்த்து உளரீதியாக திருப்தியான மனோநிலையை பெறுவதற்கு தன்னை பழக்கப்படுத்திக்கொள்ள முயற்சிக்க வேண்டுமென இ இஸ்லாம் வழிகாட்டுகிறது. 


\section{IV. இழவிரக்கம் அல்லது மரணத் துயர் (Grief)}

உளவியல் பிரச்சினைகளில் இழவிரக்கம் அல்லது மரணத் துயர் முக்கிய ஒன்றாகும். பெரும்பாலும் இது அன்புக்குரியவரின் மறைவினைத் தொடர்ந்து ஏற்படுவதாகும். இது எல்லோருக்கும் ஏற்படும் சாதாரண நிகழ்வாக இருப்பினும் சிலரில் இதன் தாக்கம் அசாதாரண நிலையினை அடையலாம் [16].

வாழ்க்கையில் இழப்புகள் இயற்கையாயினும் அவற்றை தங்கிக்கொள்ள முடியாமல் உள ரீதியாக மனிதன் விரக்திக்கு உள்ளாகிறான். எனினும் இஸ்லாமிய நோக்கில் மனித வாழ்வில் தோன்றும் இன்பம்,துன்பம், வலிகள் மற்றும் இழப்புகள் என அனைத்தும் மனிதனை சோதிக்கவென இறைவனின் நாட்டப்படி இடம்பெறுபவை ஆகும். எனவே, பொறுமையோடும் சகிப்புத்தன்மையோடும் அவற்றை எதிர்கொள்வது அவனது கடமையாகும். இதனையே அல்குர்ஆன்

“நிச்சயமாக நாம் உங்களை ஓரளவு அச்சத்தாலும், பசியாலும், பொருள்கள், உயிர்கள்,

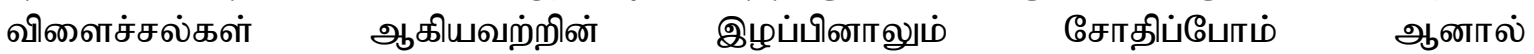
பொறுமையுடையோருக்கு (நபியே!) நீர நன்மாராயங் கூறுவீராக!, (பொறுமை உடையோராகிய) அவர்களுக்குத் துன்பம் ஏற்படும் போது, “நிச்சயமாக நாம் அல்லாஹ்வுக்கே உரியவர்கள் நிச்சயமாக நாம் அவனிடமே திரும்பிச் செல்வோம்" என்று கூறுவார்கள்" (02: 155,156) எனக் குறிப்பிடுகிறது.

இதனால் மனிதனுக்கு அவ்வப்போது ஏற்படும் வலிகளை உணர்ந்து அவற்றுக்கென வருந்துவதை இஸ்லாம் தடுக்கவில்லை. நபி (ஸல்) அவர்கள் கூட தனது மகன் இப்ராஹீமின் மரணத்தினால் கவலையுற்று கண்ணீர் வடித்ததாக (புஹாரி, 1303) நபிமொழியொன்று எமக்கு தெளிவுபடுத்துகின்றது. எனினும் எமக்கு ஏற்பட்ட துயரத்தினால் நம்மை நாமே கஷ்டப்படுத்தி அதனூடாக உளவியல் நோய்கள் ஏற்படுமளவு வருந்துவதை இஸ்லாம் வரவேற்கவில்லை என்பதே நிதர்சனமாகும்.

\section{முடிவுரை (Conclusion)}

மனித நலனைக் காக்கவென இறைவனால் அருளப்பட்ட இஸ்லாத்தின் முதன்மை மூலாதாரங்களான அல்குர்ஆன் மற்றும் அஸ்ஸுன்னா என்பன உளவளத்துணை தொடர்பில் மிக அழகிய வழிகாட்டல்களை வழங்குகின்றன. இவ்வழிகாட்டல்கள் மனிதனின் உடல், உள, ஆன்மீக மற்றும் லௌகீக தேவைகளுக்கு மத்தியில் சமநிலையை பேணுவதன் ஊடாக உள நோய்களில் இருந்து பாதுகாப்புப்பெற்று உள நிம்மதியைப்பெற வழிகாட்டுகின்றன. இஸ்லாமிய அடிப்படை மூலாதாரங்களில் உளவளத்துணை தொடர்பில் குறிப்பிடப்பட்டுள்ள கோட்பாட்டு ரீதியான பல்வேறு விடயங்கள் நபி (ஸல்) அவர்கள் மூலம் வெற்றிகரமாக நடைமுறையில் பிரயோகிக்கப்பட்டமையை பல்வேறு சான்றுகள் உறுதிப்படுத்துகின்றன. ம மதம்சார் உளவளத்துணை (Religious Counselling) தொடர்பில் பல்வேறு ஆய்வுகள் மேற்கொள்ளப்படும் நவீன உலகில் இஸ்லாமிய உளவளத்துணை வழிகாட்டல்கள் தொடர்பான கோட்பாட்டு மற்றும் நடைமுறைசார் ஆய்வுகள் மேற்கொள்ளப்படுவது இஸ்லாமிய உளவளத்துணையின் பிரயோகத்தின் முக்கியத்துவத்தை தெளிவுபடுத்துவதற்கான வழிகாட்டியாக அமையவல்லதாகும்.

\section{References}

\section{Basic Islamic Sources}

[1]. Holy Qur'an (Translation of the Meanings of The Noble Quran in the Tamil). (n.d.). Madinah, K.S.A: King Fahd Complex for the Printing of the Holy Qur'an.

[2]. Bukhārī, M. I. (2001). Sahih Bukhari. Beirut: Dar Turuk al-Najah.

[3]. Muslim ibn al-Hajjaj. (2000). Sahih Muslim. Riyadh: Dar Taiba.

[4]. Abū Dawud. (2009). Sunan Abu Dawud. Dar al-Risala.

[5]. Tirmidhī, Muhammad bin 'İsá. (1984). Jami al-Tirmidhi. Lebanon: Dar al-Kitab al-Arabi.

[6]. Ibn Mājah, Muḥammad ibn Yazīd. (1997). Sunan Ibn Majah. Riyadh: Darussalam.

[7]. Ahmad ibn Hanbal. (1993). Musnad Ahmad ibn Hanbal. Beirut: Mu'assat al-Risala. 


\section{Other Sources}

[1].Post, B.C., \& Wade, N.G. (2009). Religion and spirituality in psychotherapy: a practice-friendly review of research. Journal of Clinical Psychology 65(2),131-46. https://doi.org/10.1002/jclp.20563.

[2].Hamjah, S.H., \& Akhir, N.S.M. (2014). Islamic Approach in Counseling. Journal of Religion and Health 53(1), 279-289. https://doi.org/10.1007/s10943-013-9703-4

[3]. Weatherhead, S., \& Anna, D. (2015). Key Issues to Consider in Therapy with Muslim Families. Journal of Religion and Health 54(6), 2398-2411. https://doi.org/10.1007/s10943-015-0023-8

[4]. Abu hindi, Wayil. (2006, October 15). Al-Quran 'afdal eilaj li al-amrad alnafsiat wa al-easabia [The Qur'an is the best treatment for mental and neurological diseases]. Al-Bayan.

https://www.albayan.ae/sports/2006-10-15-1.953908

[5].Zayed, T.M. (2014). Conceptual and Practical Understanding of Counseling in Islam. Malaysian Online Journal of Counseling, 2(1), 15-27. https://mojc.um.edu.my/article/view/5560

[6]. Baqutayan, S. M. S. (2011). An Innovative Islamic Counseling. International Journal of Humanities and Social Science, 1(21), 178-183.

http://www.ijhssnet.com/journals/Vol_1_No_21_Special_Issue_December_2011/21-1.pdf

[7]. Baheej, R. (2012). Ilm al-nafs wa al-Quran al-karim [Psychology and the Holy Quran]. Alukah. https://www.alukah.net/culture/0/48150

[8]. Abdullah, M.M.A. (2020). Islāmiya uḷavalattuṇai: Ōr arimukam [Islamic Counselling: An Introduction]. Ayvukkural, 2(1), 13-21.

[9]. Rassool, G.H. (2016). Islamic Counselling an Introduction to theory and practice. UK: Routledge. https://doi.org/10.4324/9781315694993

[10].El-Aswad, E.S. (2017) Islamic Care and Counseling. In: D.A. Leeming (ed.), Encyclopedia of Psychology and Religion. Berlin, Heidelberg: Springer. https://doi.org/10.1007/978-3-642-27771-9_200074-1

[11]. Abdullah, M.M.A., \& Mazahir, S.M.M. (2021). Ārruppațuttalil paḷlivācal imāmkạ̣in vakipākam [The Role of Mosque Imams in Counselling]. KALAM-International Journal, 14(1), 121- 130. http://seu.ac.lk/fac/kalam/publication/vol14/issue1/11.\%20K2021\%20(121-130).pdf

[12]. Hallen, B. (2002). A Short History of African Philosophy. Indiana University Press.

[13]. Saadat, M.F.M. (2014). Al-Irshad al-Nafsi al-Diyni fi Daw' al-Quran al-Karim wa Sunat al-Nabawiat al-Sharifa [Religious psychological counseling in the light of the Holy Qur'an and the noble Prophet's Sunnah]. Author. https://www.alukah.net/library/0/78045

[14]. Sudan, S. A. (2017). Principles of Islamic Counselling and Psychotherapy. Asian Journal of Management Sciences \& Education, 6(3), 129-138. http://irep.iium.edu.my/id/eprint/62402

[15]. Sharkavi, H.S. (1984). Nahwa ilm nafs Islami [Towards an Islamic psychology]. Iskandariya: General Egyptian Book Organization.

[16]. Mursy, K.I., \& Rasheedi, B. (1984). Al-tawjih wa al-irshad falsafatuh wa akhlaqiatuh fi al-mujtamaat al-Islamia [Guidance and counseling, its philosophy and ethics in Islamic societies]. The Educational Journal, 1(2), 9-27. http://pubcouncil.kuniv.edu.kw/joe/homear.aspx?id=8\&Root=yes\&authid=709

[17]. Inayat, Q. (2001) The relationship between integrative and Islamic counselling. Counselling Psychology Quarterly, 14(4), 381-386. https://doi.org/10.1080/09515070110101478

[18]. Abdullah, S. (2007). Islam and counselling: Models of practice in Muslim communal life. Journal of Pastoral Counselling, 42, 42-55.

[19]. Arberry, A.J. (2001). Sufism: An Account of the Mystics of Islam. New York, United States: Dover Publication.

[20]. Jafari, Mumtaz F. (1993). Counseling Values and Objectives: A Comparison of Western and Islamic Perspectives. American Journal of Islamic Social Sciences, 10(3), 326- 339. https://doi.org/10.35632/ajis.v10i3.2490 
[21]. Aboo Hasboo, S.M.M. (2018). Asalib al-Irshad al-nafsii fi al-hadith alnabavi [Methods of psychological counseling in the Hadith]. E-Proceeding of 3rd INHAD International Muzakarah \& Mu'tamar on Hadith (IMAM 2018). 27 December 2018. KUIS,Kajang, Selangor. pp. 422-441. http://conference.kuis.edu.my/imam/images/eprosiding/2018/ba/3013-imam-2018.pdf

[22]. Sabry, W.M., \& Vohra, A. (2013). Role of Islam in the management of Psychiatric disorders. Indian Journal of Psychiatry, 55(6), 205-214. https://doi.org/10.4103/0019-5545.105534

[23]. Ramees, A. (2018). Ilankkaiyil tarkolaiyin pōkkum savālkalum [The trend of suicide in Sri Lanka and challenges]. Journal of social review, 5(1), 64-75. http://ir.lib.seu.ac.lk/handle/123456789/4222

[24]. Razak, A.L.A. (2014). Obsessive-Compulsive Disorder: Its What and How from an Islamic Perspective. Global Journal Al-Thaqafah 4(1), 7-15. DOI: 10.7187/GJAT502014.04. 